$\mathbb{T}$ periodica polytechnica

\author{
Transportation Engineering \\ $39 / 2(2011) 77 \sqrt{81}$ \\ doi: 10.3311/pp.tr.2011-2.05 \\ web: http://www.pp.bme.hu/tr \\ (c) Periodica Polytechnica 2011
}

RESEARCH ARTICLE

\section{Model-based LQ control design of integrated vehicle tracking systems}

\author{
Balázs Németh / Péter Gáspár
}

Received 2010-09-07

\begin{abstract}
The paper comprises the design of an integrated vehicle system including powertrain, brake, active suspension and steering components. The purpose is to design an integrated adaptive cruise control system with the aim of keeping the distance and tracking the path. In the first step, the vehicle model which is able to handle the effects of road and wind disturbances is formalized. In the second step, an adaptive cruise-control system based on the linear quadratic (LQ) method is designed with special emphasis on path tracking and predefined distance considerations. In the control design, several vehicle components are integrated, thus the controller of the vehicle systems operate simultaneously in one algorithm. The reason for using a linear quadratic method is to achieve a balance between different performance specifications and design an optimal control with the minimization of a cost function. During the simulations Matlab/Simulink and CarSim are used,
\end{abstract}

\section{Keywords}

integrated vehicle control $\cdot$ adaptive cruise controller $\cdot L Q$ control design $\cdot$ powertrain $\cdot$ steering $\cdot$ brake $\cdot$ active suspension.

\section{Acknowledgement}

The work is connected to the scientific program of the 'Development of quality-oriented and harmonized $R+D+I$ strategy and functional model at BME' project. This project is supported by the the Hungarian Scientific Research Fund (OTKA) through grant No. CNK-78168 and by the New Szeéchenyi Plan (Project ID: TÁMOP-4.2.1/B-09/1/KMR-2010-0002) which are gratefully acknowledged.

\section{Balázs Németh}

Department of Control and Transport Automation, BME, H-1111 Budapest, Bertalan L. u. 2, Hungary

e-mail: nemeth.balazs@mail.bme.hu

\section{Péter Gáspár}

Systems and Control Laboratory, Computer and Automation Research Institute, H-1111 Budapest, Kende u. 13-17, Hungary

e-mail: gaspar@sztaki.hu

\section{Introduction and motivation}

The growing number of electrical components poses a significant problem in terms of their phasing and communication. The problem has not only electrical reasons but it also stems from the necessity that individual control systems must work in cooperation. The industry solves this problem with CAN, LIN, Flex-Ray and other communication buses and switching, which cause mathematical and electrical difficulties. Another possibility is using integrated vehicle control systems. In this case the different control systems of the vehicle have one common control unit which controls the systems to be harmonized. It means not only one micro-controller, but also one control algorithm in it, which is able to control alone several systems belonging to it.

Several researchers have focused on the integration of control systems. A combined use of brakes and rear-steer to augment the driver's front-steer input in controlling the yaw dynamics is proposed by [4]. A four-wheel steer and four-wheel drive (4WS/4WD) controller via feed-forward and feedback compensators is proposed by [6]. A possible integration of the brake, steering and suspension system is presented by [10]. An integrated control by using steering and suspension systems is created by [5]. A global chassis control involving an active suspension and ABS to improve road holding and passenger comfort is proposed by [8]. The adaptive cruise control is also a trafficfriendly integrated vehicle system, in which the vehicle follows a preceding vehicle along the highway at a safe distance and a desired speed. The model-based control design requires a vehicle model in which the powertrain system (motor, gear-box and retarder) and the brake are integrated, e.g. [9]. An important feature of integrated vehicle control is that it is able to adapt to changes during operation and to different fault operations. Fault scenarios are the consequence of a sensor failure, the lock failure in the actuator or a loss in effectiveness. There are also numerous papers dealing with the design of reconfigurable controls, which include FDI filters, reconfigurable controllers and reconfiguration mechanisms, see e.g. [2].

This paper focuses on the design principles of the integrated vehicle control. In the complex control system several components are taken into consideration such as the powertrain, the 
brake, the steering and the active suspension.

This paper is organized as follows: Section 2 introduces the integrated vehicle control problem and performance specifications. Section 3 contains the control-oriented vehicle model, and based on that the integrated LQ controller is designed in Section 4. Section 5 shows simulation results and the last section summarizes the achievements.

\section{Principles of integrated vehicle control}

\subsection{Integrated vehicle control}

The integration of different vehicle systems is a difficult task and it raises several questions that must be answered by controller designers. Several systems are integrated and the connections between them are described by vehicle dynamic equations. The chassis of vehicle does not have a symmetric axle in the center of mass, although it looks symmetric. This is the reason for the unequal distribution of mass. Therefore the inertial tensor of the vehicle chassis contains non-zero elements: there are interconnections between three-way dynamics (longitudinal, vertical, lateral) and between rotations (pitch, roll, yaw):

$$
\left[\begin{array}{lll}
I_{x x} & I_{x y} & I_{x z} \\
I_{y x} & I_{y y} & I_{y z} \\
I_{z x} & I_{z y} & I_{z z}
\end{array}\right]
$$

In case of heavy vehicles (such as trucks, light commercial vehicles, pick-ups) the interconnection elements (Ixy, Ixz, Iyz) can not be neglected, because the positions of charges modify the symmetrizing of the vehicle in terms of mass and inertia. In addition to this connection there are other connections between vehicle systems. During a cornering maneuver the vehicle not only rotates around its vertical axis (yaw), but due to centrifugal force also around its longitudinal axis (roll). Different brake forces are acting on the wheels, thus the vehicle can turn around the vertical axis (it also causes the previously described movements), but brake forces decrease velocity (longitudinal dynamic), and the inertial force causes pitching. By using an integrated control it is possible to handle these movements together, optimizing intervention into vehicle dynamics and harmonizing safety and stability requirements.

Integrated control design has another advantage. Using a high number of independent controllers makes it difficult to harmonize the network communication and the stability of controlled systems. An integrated controller could help in this problem, because controllers of the most important (in terms of vehicle dynamic) actuators are integrated in one hardware and software unit, so switching among safety controllers is not necessary.

\subsection{Performance specifications}

The purpose of the control design is to create a complex controller. An integrated control system is designed in such a way that the effects of a control system on other vehicle functions are taken into consideration in the design process by selecting the various performance specifications.
The electric supplies of road vehicles are finite, therefore it is impossible to consume arbitrary quantities. In case of braking and driving there is a limit to the energy available, such as the engine power and active suspension forces. Vehicle body-wheel distance and tyre load are concerned with the road-holding capacity and stability [3]. In cornering the chassis of the vehicle rolls and it causes outside wheel compression and inside dissension in the suspension system. Vertical acceleration has importance in traveling comfort, in the determination of traveling comfort vertical acceleration deviation is considered using a suitable weight depending on the frequency. A decrease in vertical acceleration is also important to protect distributed cargo and stress growth on machine elements. The tracking of a reference signal (speed, distance from another vehicle, trajectory) and disturbance tolerance and correction are essential. By increasing the accurate of tracking the overrun of distance change decreases, and the controlling speed (frequency) may grow.

Achieving sufficient harmony among these criteria - mainly by using small control energy - is very hard because of the conflicts between them. Holding tracking speed or distance accurately enough and reacting disturbances fast require high control energy. By decreasing the vertical acceleration of the vehicle body (which means increasing traveling comfort) road holding will also deteriorate, so the vehicle body-wheel distance will increase. It is necessary to find a control algorithm by which these conflicting quality performances could be ensured economically.

The linear quadratic (LQ) controller can efficiently consider jointly the different quality criteria by using weight numbers. In LQ control theorem a cost function is determined, which contains quality performances and input powers [11]:

$$
\begin{aligned}
J & =\lim _{T \rightarrow \infty} \frac{1}{2} \int_{0}^{T} \sum q_{i} k_{i}^{2} d t= \\
& =\lim _{T \rightarrow \infty} \frac{1}{2} \int_{0}^{T}\left[\begin{array}{ll}
x^{T} & u^{T}
\end{array}\right]\left[\begin{array}{cc}
Q_{c} & N_{c} \\
N_{c}^{T} & R_{c}
\end{array}\right]\left[\begin{array}{l}
x \\
u
\end{array}\right] d t
\end{aligned}
$$

where $k_{i}$ formalizes a performance signal as a function of the state variables and $q_{i}$ is its weighting factor.

By minimizing the cost function the quality performances and input powers can be taken into consideration. Using $Q_{c}, R_{c}$ and $N_{c}$ weights a balance between different performance specifications can be achieved. The purpose of the control design is the calculation of an optimal state-feedback gain $K$. The optimal input signal is $u=-K x$.

The following performances are taken into consideration:

- Chassis dynamic performances: $\ddot{z}_{2}$ : vertical acceleration of the sprung mass, $\ddot{\theta}$ : pitch acceleration of the sprung mass, $\ddot{\phi}$ : roll acceleration of the sprung mass.

- Wheel dynamic performances: $F_{\text {din }_{i}}$ : dynamic loads of the wheels, $d_{i}$ : relative distance between wheel and body.

- Tracking performances: $\Delta x$ : distance or speed change depending on the control purpose, $\psi$ : tracking of yaw rate. 
- Actuators: $F_{a k t_{i}}$ : forces of suspension actuators, $F_{\text {long }}$ : longitudinal forces (drive and brake), $\Delta F_{\text {brake }}$ : difference between the brake force, $\delta$ : steering angle.

\section{Vehicle model}

The nonlinear vehicle model is defined in [7, 12]. To use model for control purpose is necessary to take some assumptions, e.g. the tyre model is necessary to linearize, velocity is considered constant in control design.

The model of a vehicle is a five-mass (four wheels and chassis) nonlinear model, see Fig. 1, $m_{s}$ is the mass of the chassis and $m_{2 i j}$ is the unsprung mass. The chassis in the model disposes 4 freedoms, vertical displacement of the center of gravity $\left(z_{s}\right)$, pitch angle $(\theta)$, roll angle $(\varphi)$ and yaw angle $(\psi)$. In the dynamic equations the pitch, roll and yaw dynamics, and inertias $I_{\theta}, I_{\varphi}, I_{\psi}$ are considered. The force elements in suspension system are the damper forces, the sprung forces and the active suspension forces. $F_{t r}, F_{w}, \Delta F_{b i}$ are the tractive force, the side-wind force, and the brake force difference on the axle, respectively. The disturbances of the model are road excitations $w_{i j}$ and wind forces. In the equations the front wheel steering is denoted by $\delta_{f}$. The vertical displacement of unsprung masses (wheels) are considered.

The state space presentation of the model is based on the next 6-states vector $x=\left[\begin{array}{llllll}\dot{\theta} & \dot{\varphi} & \beta & \dot{\psi} & \dot{z}_{s} & \dot{d}\end{array}\right]^{T}$. The states are the suspension compressions at all four suspensions, wheel rotational speeds, the vertical acceleration of the chassis, pitch rate of the chassis, and the yaw rate of the vehicle. The state space representation is as follows:

$$
\begin{aligned}
& \dot{x}=A x+B u \\
& y=C x
\end{aligned}
$$

where $A, B$ and $C$ are system matrices.

\section{Integrated adaptive cruise control design}

In order to solve an adaptive cruise control problem two vehicles are used. The model contains two vehicles, the second one tracks the leader vehicle, the roles of the second vehicle are holding distance (with a predefined threshold) and tracking the yaw of the first vehicle [6]. With an integrated cruise control system it is also possible to track a predefined trajectory. In both cases the part of the task is to generate a suitable reference signal. Another performance is to limit the input forces, because brake and traction forces have maximum values (determined by the construction of the braking system and the powertrain). Moreover, the longitudinal forces have a maximal value, which depends on the wheel-road contact. Furthermore, the force of the suspension actuator and the angle of the steering have maximal values. These features must be taken into consideration in the control algorithm. The scheme of the elementary model is shown in Fig. 2 .

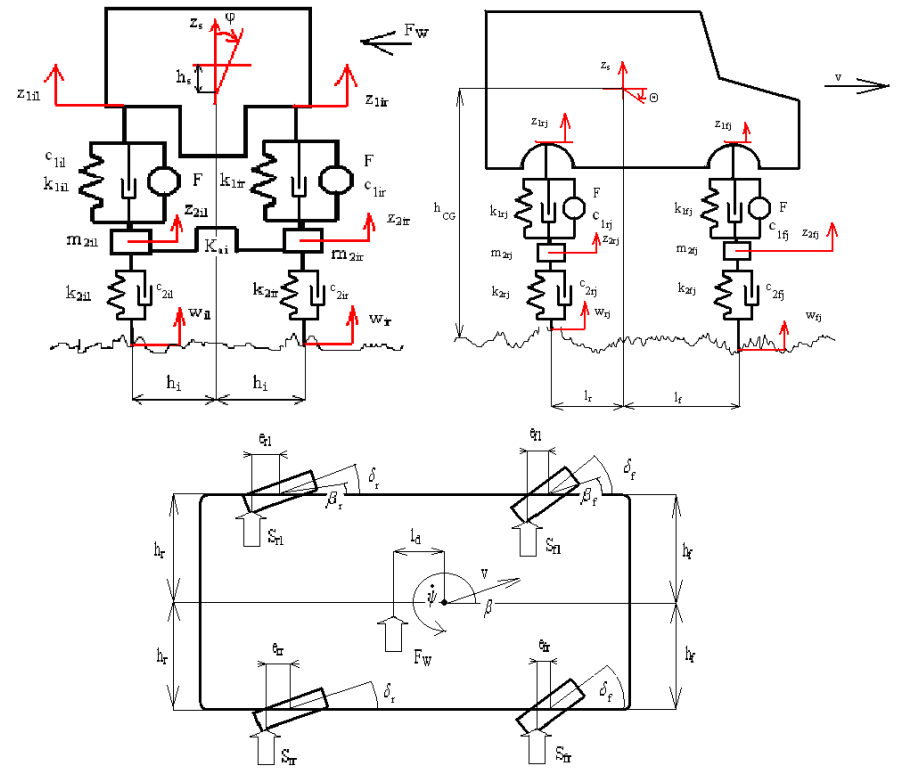

Fig. 1. Suspension model

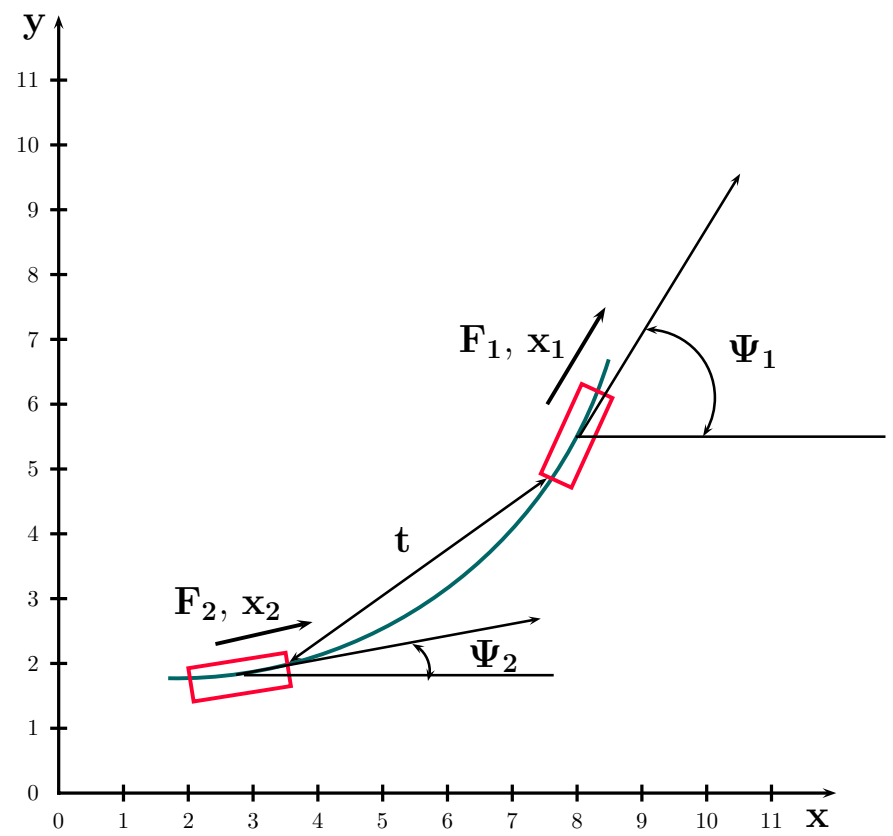

Fig. 2. Tracking model

The linear dynamical equations of the model are as follows:

$$
\begin{aligned}
& F_{1}=m_{1} \ddot{x}_{1} \\
& F_{2}=m_{2} \ddot{x}_{2}
\end{aligned}
$$

where $m_{i}$ is the mass of vehicles, $x_{i}$ is relative distance between vehicles, and $F_{i}$ is the tracking/braking force of vehicles.

These equations can be transformed into the state-space representation form by using the following expressions:

$$
\begin{aligned}
& \dot{d}=\dot{x}_{1}-\dot{x}_{2} \\
& \ddot{d}=\ddot{x}_{1}-\ddot{x}_{2}=\frac{1}{m_{1}} F_{1}-\frac{1}{m_{2}} F_{2}
\end{aligned}
$$


Finally, the state space representation is:

$$
\dot{x}=\left[\begin{array}{ll}
0 & 1 \\
0 & 0
\end{array}\right] x+\left[\begin{array}{c}
0 \\
\frac{1}{m_{1}}
\end{array}\right] F_{1}+\left[\begin{array}{c}
0 \\
-\frac{1}{m_{2}}
\end{array}\right] F_{2}
$$

where $x=\left[\begin{array}{ll}d & \dot{d}\end{array}\right]^{T}$ is the state vector with the relative distance $d=x_{1}-x_{2}, F_{1}$ is disturbance, and $F_{2}$ is the control signal. The model which is the basis of the control design is a combination of the two submodels (3) and (9).

The purpose is to ensure that the system output follows a reference command signal with an acceptable error. The difficulty of the servo control design is that the state vector does not include the output variable. Thus, there is no error information in the state feedback loop. In order to design servo systems in which the output signal follows the reference command signal the output variable is included in the state variables, and then the output becomes a part of the state vector, see [1].

The primary task is to follow a predefined path, i.e., the difference $\dot{\psi}_{\text {ref }}-\dot{\psi}$ will be minimal. The lateral dynamics of the vehicle is non-linear and the road-wheel contact is uncertain, therefore the behavior of vehicle differs significantly from the nominal model. Moreover, the difference between the longitudinal velocity and the reference velocity $\dot{d}_{r e f}-\dot{d}$ will be minimal. The performance requirements must be formalized in one quadratic criterion in the following way:

$$
J=\lim _{T \rightarrow \infty} \frac{1}{T} \int_{0}^{T}\left\{q_{1}\left(\dot{\psi}_{r e f}-\dot{\psi}\right)^{2}+q_{2}\left(\dot{d}_{r e f}-\dot{d}\right)^{2}+r \cdot \delta^{2}\right\} d t
$$

where the role of the constant weights $q_{1}, q_{2}$ and $r$ is to achieve an optimal solution.

In a real controlled system the control unit uses measured signals. In case of full state-feedback control the controller uses all states to determine control inputs. This solution requires that all the state variables be measured or available. In practice usually not all the state variables are measured, e.g. the lateral slip $\beta$. Moreover, sensors are expensive and require communications. Thus it is necessary to design an observer which is able to estimate the states by using the measured signals. The observer can also be designed by using the linear quadratic method. This system meets the Kalman's observability rank condition, therefore the system is observable. The measured signals of the vehicle to estimate the states are wheel-chassis relative distances at all four suspensions, wheel rotational speeds (all of the wheels) the vertical acceleration of the chassis and the yaw-rate of the vehicle.

\section{Simulation results}

Two software packages are used for the analysis of the controlled system. The control design itself is performed by using the Matlab/Simulink software. In this step the model, which is the basis of the control design, is built in Matlab and several control design methods are also implemented. The verification of the designed controller is performed by using the CarSim software. In this package the model of the actual vehicle is represented with high accuracy.

In order to illustrate the operation of the integrated adaptive cruise control a special vehicle maneuver is performed. During the maneuver the second car follows the leader with a reference distance and path, see Figs. ??(a) and ??(b). The velocity of second car also approaches that of the leader well.

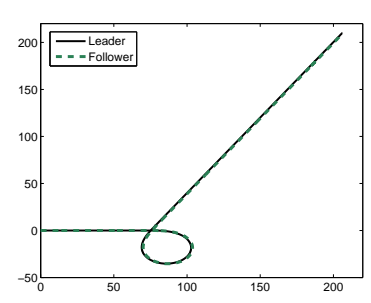

(a) Course of vehicles

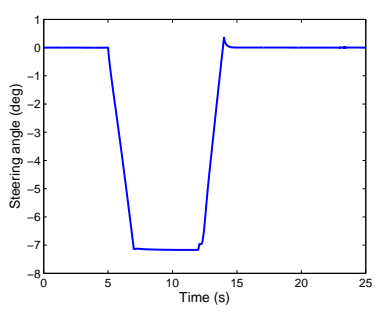

(c) Front steering angle

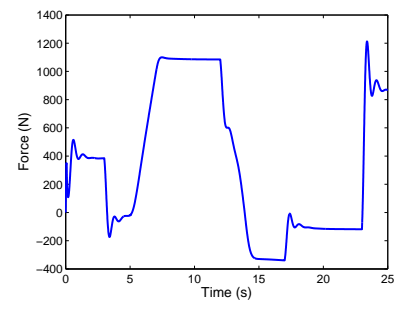

(e) Front left active suspension

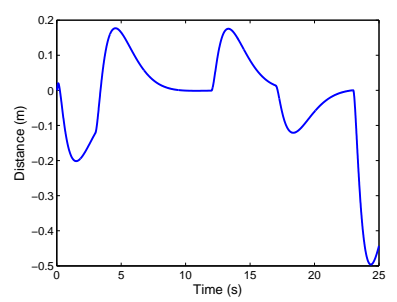

(g) Distance change between vehicles

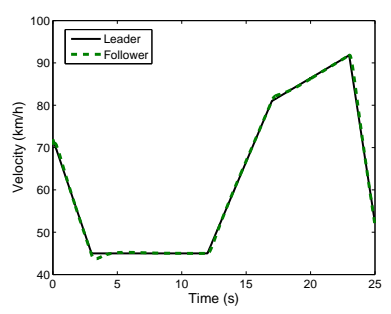

(b) Velocities of vehicles

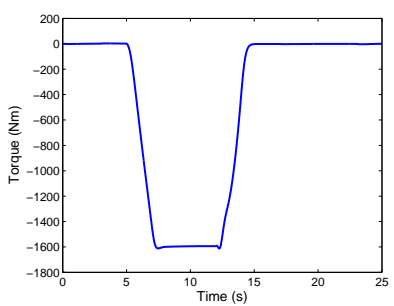

(d) Yaw torque from brake differences

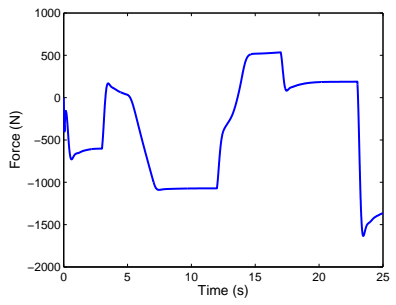

(f) Rear right active suspension

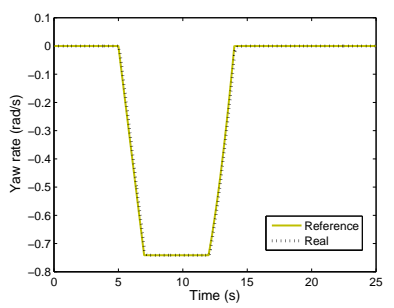

(h) Yaw rate of vehicles
Fig. 3. Illustration of the integrated vehicle control

The time responses of the front steering and the yaw torque are depicted in Fig. 3(c) and 3(d). These figures show well the efficiency of the integration: in the first part of maneuver the cornering steering angle increases to $-7^{\circ}$ and at the same time the yaw torque also increases $-1600 \mathrm{Nm}$. By increasing the velocity the follower vehicle tracks the leader one with acceptable accuracy, the change of distance between the vehicles is within tight limits, see Fig. 3. (g). Fig. 3. (h) shows that the tracking of the 
reference yaw-rate is also acceptable. The fourth integrated actuator is the active suspension. The plots of the actuated vertical forces are shown in Figs. 3 (e) and 3(f). The operation of active suspension are in a balance with the other actuators, all of the wheel-chassis distance are decreasing. It is especially important during sudden operations, e.g. in abrupt braking.

CarSim offers possibilities to simulate real vehicle tests, such as the double-lane change test. In this test the vehicle arrives into the corridor at $70 \mathrm{~km} / \mathrm{h}$ velocity and moves along without throttling. The vehicle uses an integrated controller with the front steering, the brake and active suspensions. The path of the vehicle and the yaw rate are shown in Figs. 4(a) and 4(b). The steering angle and the yaw torque operate in a harmonized way and guarantee path tracking, thus they increase the road stability of the vehicle, see Fig. 4(c) and 4(d).

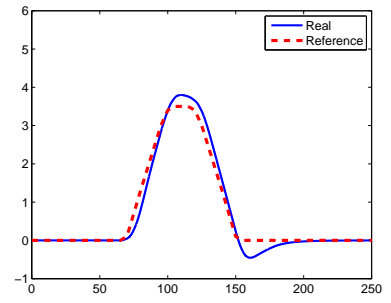

(a) Course of vehicle

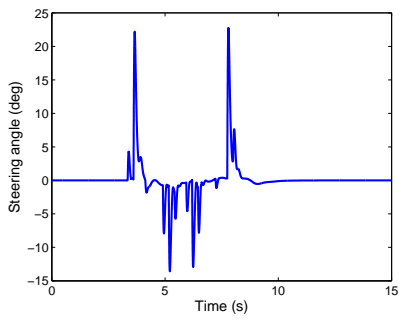

(c) Front steering angle

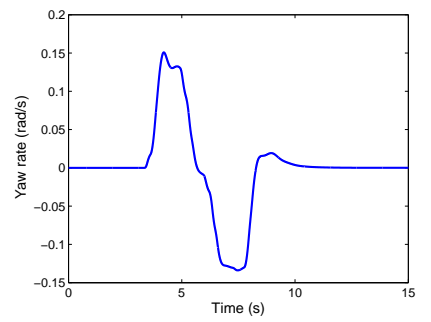

(b) Yaw rate of vehicle

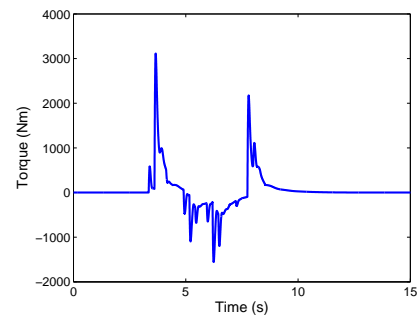

(d) Yaw torque from brake differences
Fig. 4. Double-lane change maneuver

\section{Conclusion}

In this paper an integrated adaptive cruise controller has been designed. The integrated controller designed is able to perform vehicle tracking, hold distance from an another vehicle and track its yaw rate. In addition the integrated control incorporates active suspension actuators, braking forces, traction force (throttle and gear shift) and front wheel steering. Results have been verified by simulations. Several traffic scenarios have been simulated in Matlab/Simulink and CarSim. In these simulations it has been proved that the integrated controller is able to adapt to disturbances coming from the environment and driver requirements. It can be stated that the integrated controller enhances safety and reliability in traffic.

\section{References}

Athans M, Multivarible control system, Control Lecture Note, MIT, 1985.

2 Fischer D, Isermann R, Mechatronic semi-active and active vehicle suspensions, Control Engineering Practice (2004).

3 Gordon T, An integrated strategy for the control of a full vehicle active suspension system, Vehicle System Dynamics 25 (1996), 229-242.

4 Kiencke U, Integrated vehicle control systems, Proc. of the Intelligent Components for Autonomous and Semi-Autonomous Vehicle, Tolouse, 1995, pp. 1-5.

5 Mastinu G, Babbel E, Lugner P, Margolis D, Integrated controls of lateral vehicle dynamics, Vehicle System Dynamics 23 (1994), 358-377.

6 Nagai M, Hirano Y, Yamanaka S, Intergated robust control of active rear wheel steering and direct yaw moment control, Vehicle System Dynamics 28 (1998), 416-421.

7 Németh B, Gáspár P, Vehicle modeling for integrated control design, Periodica Polytechnica (2009). submitted.

8 Poussot-Vassal C, Sename O, Dugard L, Gáspár P, Szabó Z, Bokor J, Attitude and handling improvements trough gain-scheduled suspensions and brakes control, IFAC World Congress, Seoul, 2008.

9 Rajamani R, Tan H S, Law B K, Zhang W B, Demonstration of integrated longitudinal and lateral control for the operation of automated vehicles in platoons, IEEE Transactions on Control Systems Technology, 2000, pp. 695708.

10 Trachtler A, Integrated vehicle dynamics control using active brake, steering and suspension systems, International Journal of Vehicle Design 36 (2004), $1-12$.

11 Venhovens P J T, Optimal control of vehicle suspensions. PhD Thesis, Delft University of Technology.

12 Yoshimura Y, Emoto Y, Steering and suspension system of a full car model using fuzzy reasoning and disturbance observers, International Journal Vehicle Autonomous Systems (2003). 